\title{
BALANCED METRICS ON UNIRULED MANIFOLDS
}

\author{
IONUȚ CHIOSE, RAREŞ RĂSDEACONU, AND IOANA ŞUVAINA
}

\begin{abstract}
We show that an $n$-dimensional Moishezon manifold is uniruled if and only if it supports a balanced metric $\omega^{n-1}$ of positive total scalar Chern curvature. A similar statement also holds true for class $\mathscr{C}$ manifolds of dimension three.
\end{abstract}

\section{Contents}

Introduction

1. Total scalar curvatures

2. Positive cones in Bott-Chern and Aeppli cohomology groups 5

3. Uniruled manifolds and balanced metrics 12

4. Balanced metrics on twistor spaces 16

References

\section{INTRODUCTION}

A compact complex manifold $M$ is called uniruled if there exists a rational curve passing through every point of $M$. A differential geometric characterization of uniruledness in complex dimension two was given by Yau [Ya]. He proved that a Kähler surface $S$ has Kodaira dimension $-\infty$ (equivalently, uniruled) if and only if it admits a Kähler metric $\omega$ of positive total scalar curvature. This is equivalent to

$$
\int_{S} c_{1}\left(K_{S}\right) \wedge \omega<0
$$

where $K_{S}$ denotes the canonical line bundle of $S$.

The aim of this article is to extend Yau's differential geometric characterization in higher dimensions. In one direction, the existence of a Kähler metric of positive total scalar curvature on projective uniruled manifolds has been recently discussed by Heier and Wong [HW, Section 5], but a definite conclusion is elusive. Such metrics are known to exist on some uniruled manifolds. Most notably, they exist on projective Mori fiber spaces of dimension three, as established by Demailly, Peternell and Schneider [DPS, Proposition 4.9]. An approach to this existence question, which indicates that in general the answer is negative, is proposed by the second author in the case of rationally connected threefolds [Ră]. This suggests

Date: October 11, 2016.

2000 Mathematics Subject Classification. Primary: 53C55, 32Q10; Secondary: 32J18, 14E30, $14 \mathrm{M} 99$.

Key words and phrases. Complex manifolds, uniruledness, balanced metrics, total scalar Chern curvature. 
that instead of searching for Kähler metrics of positive total scalar curvature on uniruled manifolds, one should broaden the search to a larger class of metrics. To detect a suitable such class of Hermitian metrics we follow Yau's original proof [Ya]. Yau's approach to find Kähler metrics of positive total scalar curvature on uniruled surfaces relies on the minimal model theory. His proof follows in two steps:

A) Bimeromorphic invariance: Yau shows that the existence of such metrics is an invariant property under bimeromorphic maps. In the case of surfaces, the invariance under blow-ups suffices.

B) Existence of a Kähler metric of positive total scalar Chern curvature on an exhaustive list of bimeromorphism classes of uniruled surfaces: Yau proved the existence of Kähler metrics satisfying (0.1) on all geometrically ruled surfaces.

To extend Step A in higher dimensions, recall that any bimeromorphic map decomposes by the weak factorization theorem [AKMW, Theorem 0.3.1] into a sequence of blow-ups and blow-downs with smooth centers. A well-known fact is that, unlike uniruledness, the class of Kähler manifolds of dimension greater than or equal to three is not closed under bimeromorphisms. We are led to consider a larger class of manifolds which is invariant under bimeromorphisms. From the work of Alessandrini and Bassanelli [AB1, AB2], it is known that the class of manifolds carrying balanced metrics, i.e., Hermitian metrics with co-closed Kähler form (see [Mi] and Section 1), satisfies this property. In dimension two, any balanced metric is in fact Kähler, but in higher dimensions there exist non-Kähler manifolds which admit balanced metrics or Kähler manifolds which admit non-Kähler balanced metrics. We prove:

Theorem A. Let $X$ and $Y$ be two bimeromorphic compact complex manifolds of dimension $n$. If there exists a balanced metric $\omega_{X}^{n-1}$ on $X$ such that

$$
\int_{X} c_{1}\left(K_{X}\right) \wedge \omega_{X}^{n-1}<0
$$

then there exists a balanced metric $\omega_{Y}^{n-1}$ on $Y$ such that

$$
\int_{Y} c_{1}\left(K_{Y}\right) \wedge \omega_{Y}^{n-1}<0
$$

Demailly, Peternell and Schneider also asked if Step A can be accomplished for normal projective varieties [DPS, Problem 4.12]. Theorem A gives a partial answer to their question.

An extension of Step B to uniruled manifolds of higher dimensions relies on the state of the art of the minimal model program. For projective uniruled manifolds one can find a bimeromorphic simpler model in any dimension [BCHM]. These bimeromorphic models are higher dimensional analogs of the geometrically ruled surfaces, called Mori fiber spaces (see Section 3.2). We show that every Mori fiber space admits Kähler metrics of positive total scalar curvature, and we obtain:

Theorem B. Every $n$-dimensional, Moishezon, uniruled manifold $X$ admits a balanced metric $\omega^{n-1}$ such that

$$
\int_{X} c_{1}\left(K_{X}\right) \wedge \omega^{n-1}<0 .
$$


Recall that a compact complex manifold is Moishezon if it is bimeromorphic to a projective manifold.

We provide two proofs for this result. One proof uses the minimal model program. A second proof is based on ideas of Toma [To], and it relies on the results of Boucksom, Demailly, Păun and Peternell [BDPP], bypassing the minimal model program.

A generalization of the minimal model program to the class of Kähler manifolds is known only in complex dimension three [HP1, HP2]. We prove the following extension of Theorem B in dimension three:

Theorem C. Every uniruled threefold $X$ of class $\mathscr{C}$ admits a balanced metric $\omega^{2}$ such that

$$
\int_{X} c_{1}\left(K_{X}\right) \wedge \omega^{2}<0
$$

Recall that a complex manifold is called of class $\mathscr{C}$ if it is bimeromorphic to a Kähler manifold. This class of manifolds is strictly larger than the class of Kähler manifolds in dimension three or more, and it contains the class of Moishezon manifolds. Every class $\mathscr{C}$ manifold carries balanced metrics by [AB1, AB2].

The bimeromorphism invariance of the class of balanced manifolds indicates that balanced metrics are natural to be considered as good replacements of Kähler metrics in order to extend Yau's differential geometric characterization of uniruledness in higher dimensions. However, this is not the only class of Hermitian metrics with such good properties. In fact, every complex manifold admits Gauduchon metrics, that is positive $(1,1)$-forms $\omega$ such that $\partial \bar{\partial} \omega^{n-1}=0$ [Ga1]. Notice that every balanced metric is a Gauduchon metric, while the converse is false. Moreover, from the positivity criterion of Lamari [La1] and [BDPP, Corollary 0.3] one can see that every uniruled projective manifold admits Gauduchon metrics of positive total scalar Chern curvature (see also Theorem D below). In Theorems B and C we prove therefore a stronger result.

Conversely, Yau's approach [Ya] can be adapted to show that the existence of a Kähler or a balanced metric of positive total scalar Chern curvature on a complex manifold implies that the Kodaira dimension of the manifold is $-\infty$. One can easily see that uniruledness implies that Kodaira dimension is $-\infty$, but the converse is a well-known open problem. Heier and Wong were able to show in [HW, Theorem 1.1] that every projective manifold which admits a Kähler metric of positive total scalar curvature is in fact uniruled. We extend here Theorem 1.1 of Heier and Wong [HW], and combining with the results from Theorems B and $\mathrm{C}$ we provide the following characterization of uniruledness:

Theorem D. Let $X$ be an n-dimensional Moishezon manifold. The following statements are equivalent:

i) $K_{X}$ is not pseudoeffective;

ii) $X$ is uniruled;

iii) $X$ admits a balanced metric of positive Chern total scalar curvature;

iv) $X$ admits a Gauduchon metric of positive Chern total scalar curvature. Moreover, the same statements hold true if $n=3$ and $X$ is of Fujiki class $\mathscr{C}$. 
The proof of the implications $i v) \Longrightarrow i) \Longrightarrow i i$ ) relies on the positivity criterion of Lamari [La1, Théorème 1.2 (1)], and on remarkable results of Boucksom, Demailly, Peternell and Păun [BDPP] and Brunella [Br].

We explore next the possibility of extending the above characterization of uniruledness in terms of the positivity of the total scalar Chern curvature of a balanced metric beyond class $\mathscr{C}$. In general, the existence of a balanced metric fails. However, in dimension three, a large class of uniruled manifolds admitting such metrics is given by complex manifolds bimeromorphic to twistor spaces [AHS]. We prove:

Theorem E. Every three dimensional complex manifold $X$ bimeromorphic to a twistor space admits a balanced metric $\omega^{2}$ such that

$$
\int_{X} c_{1}\left(K_{X}\right) \wedge \omega^{2}<0
$$

\section{Total SCALAR CURVATURES}

In this section we briefly recall some well-known background material in complex differential geometry to introduce the terminology.

Let $(M, g)$ be a Hermitian manifold and $\omega$ its Kähler form. On $(M, g)$ one can consider two canonical connections: the Levi-Civita connection, and the Chern connection.

Let $s$ denote the scalar curvature of the Levi-Civita connection. The total scalar Riemannian curvature is defined as

$$
\int_{M} s \mu_{g}=\int_{M} \frac{s \omega^{n}}{n !},
$$

where $\mu_{g}=\frac{\omega^{n}}{n !}$ is the volume form.

Let $s_{C}$ denote the scalar curvature of the Chern connection associated to the Hermitian metric $g$. The total scalar Chern curvature is defined by

$$
\int_{M} s_{C} \mu_{g}
$$

The Ricci curvature form of the Chern connection represents the first Chern class of $M$ rescaled by a factor of $2 \pi$, and $c_{1}(M)=-c_{1}\left(K_{M}\right)$, where $K_{M}$ is the canonical line bundle of $M$. Since the scalar curvature is the trace of the Ricci curvature form, we can write

$$
\int_{M} s_{C} \mu_{g}=\int_{M} \frac{s_{C} \omega^{n}}{n !}=-\frac{2 \pi}{(n-1) !} \int_{M} c_{1}\left(K_{M}\right) \wedge \omega^{n-1} .
$$

A result due to Gauduchon [Ga2, page 506] (see also [LY, Corollary 1.11]) compares the total scalar Riemannian curvature and the total scalar Chern curvature:

Proposition 1.1. Let $(M, g)$ be a compact, complex manifold equipped with a Hermitian metric. Then

$$
\int_{M} s_{C} \mu_{g} \geq \frac{1}{2} \int_{M} s \mu_{g},
$$

with equality if and only if the metric is Kähler. 
Corollary 1.2. Let $(M, g)$ be a compact, complex manifold of dimension $n$ equipped with a Hermitian metric. If the scalar Riemannian curvature of $M$ is positive, then

$$
\int_{M} c_{1}\left(K_{M}\right) \wedge \omega^{n-1}<0 .
$$

Definition 1.1. Let $(M, g)$ be a compact complex manifold of complex dimension $n$ equipped with a Hermitian metric $g$, and let $\omega$ denote its Kähler form. If $d \omega=0$, then $g$ is called a Kähler metric. A complex manifold which admits a Kähler metric is called a Kähler manifold.

If $g$ is a Kähler metric, then its Kähler form $\omega$ is a real, $d$-closed, strictly positive $(1,1)$-form. Conversely, given a smooth, strictly positive, $d$-closed $(1,1)$-form $\omega$, there exists a Hermitian metric $g$ whose Kähler form is $\omega$. We will use the notation $(M, \omega)$ to denote a Kähler manifold with prescribed Kähler form.

Definition 1.2. Let $(M, g)$ be a compact complex manifold of complex dimension $n$ equipped with a Hermitian metric $g$, and let $\omega$ denote its Kähler form. If $d\left(\omega^{n-1}\right)=$ 0 , then $g$ is called a balanced metric. A complex manifold which admits a balanced metric is called a balanced manifold. We will use the notation $\left(M, \omega^{n-1}\right)$ to denote a balanced manifold.

Given a balanced metric of Kähler form $\omega$, the $(n-1, n-1)$-form $\omega^{n-1}$ is real, strictly positive and $d$-closed. Conversely, it is an easy exercise in linear algebra to see that given a real, strictly positive, $d$-closed $(n-1, n-1)$-form $\Omega$, there exists a unique Hermitian metric of Kähler form $\omega$ such that $\Omega=\omega^{n-1}$ ([Mi, page 279]). Throughout the paper, by a balanced metric we mean a real, $d$-closed, strictly positive $(n-1, n-1)$-form, denoted by $\omega^{n-1}$.

A Kähler manifold is balanced, and if $n=2$ the converse is also true. In higher dimensions the converse is false. A large class of counterexamples is provided by the twistor spaces of closed anti-self-dual four-manifolds (see Sect. 4). Another interesting class of non-Kähler balanced manifolds has been found by $\mathrm{Fu}, \mathrm{Li}$ and Yau. In [FLY], the authors showed that the complex structures with trivial canonical bundles constructed by Lu and Tian [LT] and Friedman [Fr] on connected sums of $S^{3} \times S^{3}$ carry a balanced metric.

\section{Positive cones in Bott-Chern and Aeppli cohomology groups}

In this section we recall the definitions of the Bott-Chern and Aeppli cohomology groups, and of the pseudoeffective and the nef cones. In the Kähler case, these cohomology groups are isomorphic to the usual Dolbeault cohomology groups due to the $\partial \bar{\partial}$-lemma. However, we prefer to work with the Bott-Chern and Aeppli cohomology groups since the class of a $d$ - or $i \partial \bar{\partial}$-closed positive current lies naturally in these cohomology groups, and, moreover, the duality statements between the nef and pseudoeffective cones (Theorem 2.4) can be naturally stated in this setting. For more details, see $[\mathrm{Sc}]$.

Let $X$ be a compact complex manifold of dimension $n$. The Bott-Chern cohomology groups are defined as

$$
H_{B C}^{p, q}(X, \mathbb{C})=\frac{\left\{\alpha \in \mathscr{C}_{p, q}^{\infty}(X) \mid d \alpha=0\right\}}{\left\{i \partial \bar{\partial} \beta \mid \beta \in \mathscr{C}_{p-1, q-1}^{\infty}(X)\right\}},
$$


and the Aeppli cohomology groups are

$$
H_{A}^{p, q}(X, \mathbb{C})=\frac{\left\{\alpha \in \mathscr{C}_{p, q}^{\infty}(X) \mid i \partial \bar{\partial} \alpha=0\right\}}{\left\{\partial \beta+\bar{\partial} \gamma \mid \beta \in \mathscr{C}_{p-1, q}^{\infty}(X), \gamma \in \mathscr{C}_{p, q-1}^{\infty}(X)\right\}}
$$

Since all the operators involved in the definitions of the above cohomology groups are real in bidegrees $(p, p)$ the real cohomology groups $H_{B C}^{p, p}(X, \mathbb{R})$ and $H_{A}^{p, p}(X, \mathbb{R})$ are well-defined. The above groups can be defined by using smooth forms or currents. We use the notation $[s]$ for the class of a $d$-closed form or current $s$ in $H_{B C}^{\bullet \cdot \bullet}$ and $\{t\}$ for the class of a $\partial \bar{\partial}$-closed form or current $t$ in $H_{A}^{\bullet \bullet \bullet}$. The groups $H_{B C}^{p, q}(X, \mathbb{C})$ and $H_{A}^{n-p, n-q}(X, \mathbb{C})$ are dual via the pairing

$$
H_{B C}^{p, q}(X, \mathbb{C}) \times H_{A}^{n-p, n-q}(X, \mathbb{C}) \rightarrow \mathbb{C},([\alpha],\{\beta\}) \rightarrow \int_{X} \alpha \wedge \beta
$$

By an abuse of notation, we also denote by $(\alpha, \beta)$ the evaluation $\int_{X} \alpha \wedge \beta$, regardless of whether $\alpha$ and $\beta$ denote appropriate forms, currents or cohomology classes.

Definition 2.1 (Lelong [Le]). Let $T$ be a current of bi-dimension $(p, p)$. We say that $T$ is a positive current, and we write $T \geq 0$, if $T \wedge i \alpha_{1} \wedge \bar{\alpha}_{1} \wedge \cdots \wedge i \alpha_{p} \wedge \bar{\alpha}_{p}$ is a positive measure, for all smooth $(1,0)$-forms $\alpha_{1}, \ldots, \alpha_{p}$.

For $\# \in\{B C, A\}$ and $p \in\{1, n-1\}$ we define the following cones:

(1) the $\#-$ pseudoeffective cone

$$
\mathscr{E}_{X, \#}^{p}=\left\{\gamma \in H_{\#}^{p, p}(X, \mathbb{R}) \mid \exists T \geq 0, T \in \gamma\right\},
$$

where by $T$ we denote here a current.

(2) the \#-nef cone

$$
\mathscr{N}_{X, \#}^{p}=\left\{\gamma \in H_{\#}^{p, p}(X, \mathbb{R}) \mid \forall \varepsilon>0, \exists \alpha_{\varepsilon} \in \gamma, \alpha_{\varepsilon} \geq-\varepsilon \omega^{p}\right\}
$$

where $\omega$ is the Kähler form of a fixed Hermitian metric on $X$ and $\alpha_{\varepsilon}$ denotes a smooth $(p, p)$-form.

Notice that all of the cones defined above are convex cones.

Remark 2.1. The pseudoeffective and nef cones $\mathscr{E}_{X, B C}^{1}$ and $\mathscr{N}_{X, B C}^{1}$ were first introduced by Demailly [De, Definition 1.3], who stressed their importance. We adapt here his definitions to $(n-1, n-1)$ Bott-Chern cohomology classes and to $(p, p)$ Aeppli cohomology classes, where $p \in\{1, n-1\}$.

The following two lemmas are standard, and some of the statements below are proved in [De, Proposition 6.1]. As they play a crucial part in our argument, and for the reader's convenience, we include their proofs.

Lemma 2.2. The cone $\mathscr{E}_{X, B C}^{1}$ is closed and $\mathscr{N}_{X, B C}^{1} \subset \mathscr{E}_{X, B C}^{1}$.

Proof. The proof of the lemma relies on the existence of Gauduchon metrics on any compact complex manifold [Ga1]. That means $X$ admits a Hermitian metric $g$ with Kähler form $\omega$ satisfying $\partial \bar{\partial} \omega^{n-1}=0$.

Indeed, suppose $\left(\left[T_{j}\right]\right)_{j}$ is a sequence of pseudoeffective classes represented by the closed positive currents $T_{j}$ such that $\left[T_{j}\right] \rightarrow \gamma \in H_{B C}^{1,1}(X, \mathbb{R})$. Fix $g$ a Gauduchon metric with Kähler form $\omega$, and notice that $\int_{X} T_{j} \wedge \omega^{n-1}$ depends only on the Aeppli cohomology class $\left\{\omega^{n-1}\right\}$ and on the BC-cohomology class $\left[T_{j}\right]$, not on the representative $\omega$. Since the sequence $\left(\int_{X} T_{j} \wedge \omega^{n-1}\right)_{j}$ is bounded, we can assume, 
after passing to a subsequence, that $\left(T_{j}\right)_{j}$ is weakly convergent to a closed positive current $T$. Then $\gamma=[T] \in \mathscr{E}_{X, B C}^{1}$.

To prove that $\mathscr{N}_{X, B C}^{1} \subset \mathscr{E}_{X, B C}^{1}$, let $[\alpha] \in \mathscr{N}_{X, B C}^{1}$, where $\alpha$ is a $d$-closed smooth $(1,1)$-form. Then, by definition, for every $\varepsilon>0$, there exists $\varphi_{\varepsilon} \in \mathscr{C}^{\infty}(X, \mathbb{R})$ such that $\alpha_{\varepsilon}:=\varepsilon \omega+\alpha+i \partial \bar{\partial} \varphi_{\varepsilon} \geq 0$. Since $\int_{X} \alpha_{\varepsilon} \wedge \omega^{n-1}$ is bounded for $0<\varepsilon \leq 1$, we extract a weakly convergent subsequence $\left(\alpha_{\varepsilon_{j}}\right)_{j}$, converging to a closed, positive current in class $[\alpha]$. Hence $[\alpha] \in \mathscr{E}_{X, B C}^{1}$.

Lemma 2.3. The cones $\mathscr{N}_{X, \#}^{p}$ are closed, where $p \in\{1, n-1\}$ and $\# \in\{B C, A\}$.

Proof. Let $\left\{\gamma_{j}\right\}_{j}$ be a sequence, where $\gamma_{j} \in \mathscr{N}_{X, \#}^{p}$ and $\gamma_{j} \rightarrow \gamma$ in $H_{\#}^{p, p}(X, \mathbb{R})$. In each cohomology class $\gamma_{j}$, we choose the unique harmonic representative $\beta_{j}$ and let $\beta$ be the unique harmonic representative in $\gamma$ with respect to some fixed Hermitian metric on $X$ (see [Sc] for more on the harmonic forms in the Bott-Chern and Aeppli cohomology groups ${ }^{1}$ ). Then, from the standard theory of elliptic operators, it follows that $\beta_{j} \rightarrow \beta$ in the $\mathscr{C}^{\infty}$ topology. This immediately implies that $\gamma$ is nef. Indeed, for every $p \in\{1, n-1\}$, given $\varepsilon>0$, we can find $j_{\varepsilon}$ such that $\beta-\beta_{j_{\varepsilon}} \geq-\frac{\varepsilon}{2} \omega^{p}$. Since $\gamma_{j_{\varepsilon}}$ (which is the class of $\beta_{j_{\varepsilon}}$ ) is nef, it follows that for every $\delta>0$ there exists a smooth form $\lambda_{\varepsilon, \delta} \in \gamma_{j_{\varepsilon}}$ such that $\lambda_{\varepsilon, \delta} \geq-\frac{\delta}{2} \omega^{p}$. Notice now that, for every $\varepsilon>0$ and $\delta>0, \beta-\beta_{j_{\varepsilon}}+\lambda_{\varepsilon, \delta}$ is a smooth representative of $\gamma$ which is $\geq-\frac{\varepsilon+\delta}{2} \omega^{p}$. Therefore $\gamma$ is nef.

Given $V$ a real vector space, denote by $V^{*}$ its dual. If $C$ a convex cone in $V$, we denote by $C^{*} \subset V^{*}$ its dual:

$$
C^{*}=\left\{v^{*} \in V^{*} \mid v^{*}(c) \geq 0, \forall c \in C\right\} .
$$

By the Hahn-Banach Theorem, we have $C^{* *}=\bar{C}$.

Theorem 2.4. Let $X$ be a compact complex manifold of dimension $n$. Then

i) $\mathscr{N}_{X, B C}^{1}=\left(\mathscr{E}_{X, A}^{n-1}\right)^{*}$,

ii) $\mathscr{N}_{X, A}^{n-1}=\left(\mathscr{E}_{X, B C}^{1}\right)^{*}$.

Moreover, if $X$ is balanced, then

iii) $\mathscr{N}_{X, A}^{1}=\left(\mathscr{E}_{X, B C}^{n-1}\right)^{*}$,

iv) $\mathscr{N}_{X, B C}^{n-1}=\left(\mathscr{E}_{X, A}^{1}\right)^{*}$.

Proof. The proof of the above statements either follows directly from [La1], or the arguments in [La1] go through mutatis mutandis. For the convenience of the reader we include the details in the cases ii), iii) and iv) which are not covered by the results in [La1].

i) This is Théorème $1.2(1)$ in [La1]. ${ }^{2}$

ii) Clearly $\mathscr{N}_{X, A}^{n-1} \subset\left(\mathscr{E}_{X, B C}^{1}\right)^{*}$. Conversely, $\left(\mathscr{E}_{X, B C}^{1}\right)^{*} \subset \mathscr{N}_{X, A}^{n-1}$ is equivalent to $\left(\mathscr{N}_{X, A}^{n-1}\right)^{*} \subset \mathscr{E}_{X, B C}^{1}$ since $\mathscr{E}_{X, B C}^{1}$ is closed. Let $[\eta] \in H_{B C}^{1,1}(X, \mathbb{R})$ be such that $([\eta], \gamma) \geq 0, \forall \gamma \in \mathscr{N}_{X, A}^{n-1}$. In particular, $(\eta, \Omega) \geq 0$ for any positive

\footnotetext{
${ }^{1}$ The Bott-Chern Laplacian was introduced by Kodaira and Spencer in [KS, page 71]. In op. cit., Schweitzer adapted this construction to define the Aeppli Laplacian on the same model.

${ }^{2}$ The cones $\mathscr{N}_{X, B C}^{1}$ and $\mathscr{E}_{X, A}^{n-1}$ are the denoted by $P_{\text {nef }}^{1}(X)$ and $\Pi^{n-1}$, respectively in [La1, Théorème $1.2(1)]$.
} 
$i \partial \bar{\partial}$-closed $(n-1, n-1)$ form $\Omega$ on $X$. Lemme 1.4 in [La1] implies the existence of a distribution $\chi$ such that $\eta+i \partial \bar{\partial} \chi \geq 0$, that is $[\eta] \in \mathscr{E}_{X, B C}^{1}$.

iii) The inclusion $\mathscr{N}_{X, A}^{1} \subset\left(\mathscr{E}_{X, B C}^{n-1}\right)^{*}$ follows immediately. For the opposite inclusion, we adapt the proof of Lemme 1.3 in [La1] to our situation.

Let $\{\eta\} \in H_{A}^{1,1}(X, \mathbb{R})$ be an Aeppli cohomology class such that $(\{\eta\}, \gamma) \geq$ $0, \forall \gamma \in \mathscr{E}_{X, B C}^{n-1}$, and $\eta \in \mathscr{C}_{1,1}^{\infty}(X, \mathbb{R})$ a representative.

We proceed by fixing a Hermitian metric on $X$, with Kähler form $\phi$. Let $\mathscr{D}^{\prime n-1, n-1}(X, \mathbb{R})$ denote the space of real currents of bidegree $(n-1, n-1)$ on $X$, and define

$$
C^{n-1}=\left\{T \in \mathscr{D}^{\prime n-1, n-1}(X, \mathbb{R}) \mid T \geq 0,(T, \phi)=1\right\},
$$

which is a convex, compact set.

The set $V$ of all balanced metrics on $X$ is an open convex cone in

$$
E=\left\{\lambda^{n-1} \in \mathscr{C}_{n-1, n-1}^{\infty}(X, \mathbb{R}) \mid d \lambda^{n-1}=0\right\} .
$$

We have $\left(\eta, \omega^{n-1}\right) \geq 0, \forall \omega^{n-1} \in V$. If $\left(\eta, \omega^{n-1}\right)=0, \forall \omega^{n-1} \in V$, then $\left(\eta, \lambda^{n-1}\right)=0, \forall \lambda^{n-1} \in E$ since $V$ is open in $E$. From the duality between $H_{B C}^{n-1, n-1}(X, \mathbb{R})$ and $H_{A}^{1,1}(X, \mathbb{R})$ it follows that $\{\eta\}=0 \in \mathscr{N}_{X, A}^{1}$. We can therefore suppose that there exists $\omega_{0}^{n-1} \in V$ a balanced metric such that $\left(\eta, \omega_{0}\right)>0$. Let $D^{n-1}=C^{n-1} \cap E^{\prime}$, where

$$
E^{\prime}=\left\{T \in \mathscr{D}^{\prime n-1, n-1}(X, \mathbb{R}) \mid d T=0\right\} .
$$

It is a convex, compact subset of $\mathscr{D}^{\prime n-1, n-1}(X, \mathbb{R})$ which is non-empty, as it contains the balanced metrics. Without loss of generality, we can assume that $\omega_{0}^{n-1} \in D^{n-1}$, i.e., that $\left(\omega_{0}^{n-1}, \phi\right)=1$.

For $\varepsilon>0$, set $C(\varepsilon)=C^{n-1}+\varepsilon \omega_{0}^{n-1}$ and $D(\varepsilon)=D^{n-1}+\varepsilon \omega_{0}^{n-1}$. As $\omega_{0}^{n-1}$ is $d$-closed, we have $C(\varepsilon) \cap E^{\prime}=D(\varepsilon)$. Since $(\eta, T) \geq 0, \forall T \in D^{n-1}$ and $\left(\eta, \omega_{0}^{n-1}\right)>0$, it follows that $(\eta, T)>0, \forall T \in D(\varepsilon)$. The subspace

$$
F=E^{\prime} \cap\left\{T \in \mathscr{D}^{\prime n-1, n-1}(X, \mathbb{R}) \mid(\eta, T)=0\right\}
$$

is closed in $\mathscr{D}^{\prime n-1, n-1}(X, \mathbb{R})$ and of codimension 1 in $E^{\prime}$. Moreover,

$$
\begin{aligned}
C(\varepsilon) \cap F & =C(\varepsilon) \cap E^{\prime} \cap\left\{T \in \mathscr{D}^{\prime n-1, n-1}(X, \mathbb{R}) \mid(\eta, T)=0\right\} \\
& =D(\varepsilon) \cap\left\{T \in \mathscr{D}^{\prime n-1, n-1}(X, \mathbb{R}) \mid(\eta, T)=0\right\} \\
& =\emptyset .
\end{aligned}
$$

We can therefore separate $C(\varepsilon)$ and $F$ with a smooth $(1,1)$ form $\beta_{\varepsilon}$ which vanishes on $F$ and is strictly positive on $C(\varepsilon)$. If we let $\lambda_{\varepsilon}=\frac{\left(\eta, \omega_{0}^{n-1}\right)}{\left(\beta_{\varepsilon}, \omega_{0}^{n-1}\right)}$, then the (1,1)-form $\eta-\lambda_{\varepsilon} \beta_{\varepsilon}$ is zero on $E^{\prime}$. Therefore, from the duality between $H_{A}^{1,1}(X, \mathbb{R})$ and $H_{B C}^{n-1, n-1}(X, \mathbb{R})$, it follows that there exists $\gamma_{\varepsilon}$ a smooth $(1,0)$-form such that

$$
\eta-\lambda_{\varepsilon} \beta_{\varepsilon}=-\bar{\partial} \gamma_{\varepsilon}-\partial \bar{\gamma}_{\varepsilon}
$$

and the (1,1)-form

$$
\eta+\bar{\partial} \gamma_{\varepsilon}+\partial \bar{\gamma}_{\varepsilon}=\lambda_{\varepsilon} \beta_{\varepsilon}
$$


is strictly positive on $C(\varepsilon)$. If $T \in C^{n-1}$, then $T+\varepsilon \omega_{0}^{n-1} \in C(\varepsilon)$ and so

$\left(\eta+\bar{\partial} \gamma_{\varepsilon}+\partial \bar{\gamma}_{\varepsilon}, T+\varepsilon \omega_{0}^{n-1}\right)=\left(\eta+\bar{\partial} \gamma_{\varepsilon}+\partial \bar{\gamma}_{\varepsilon}, T\right)+\varepsilon\left(\eta, \omega_{0}^{n-1}\right)>0$.

Hence $\left(\eta+\bar{\partial} \gamma_{\varepsilon}+\partial \bar{\gamma}_{\varepsilon}, T\right)>-\varepsilon\left(\eta, \omega_{0}^{n-1}\right), \forall T \in C^{n-1}$.

Set now $m=\left(\eta, \omega_{0}^{n-1}\right)$. If $T$ is a positive non-zero current of bidegree $(n-1, n-1)$ on $X$, then $\frac{1}{(T, \phi)} T \in C^{n-1}$, therefore

$$
\left(\eta+\bar{\partial} \gamma_{\varepsilon}+\partial \bar{\gamma}_{\varepsilon}, T\right) \geq-\varepsilon m(T, \phi), \forall T \geq 0
$$

which means $\eta+\bar{\partial} \gamma_{\varepsilon}+\partial \bar{\gamma}_{\varepsilon} \geq-\varepsilon m \phi$. This implies that $\{\eta\} \in \mathscr{N}_{X, A}^{1}$.

iv) If $X$ is balanced, then $\mathscr{E}_{X, A}^{1}$ is closed (see Lemma 2.5 below). Clearly $\mathscr{N}_{X, B C}^{n-1} \subset\left(\mathscr{E}_{X, A}^{1}\right)^{*}$ and the other inclusion is equivalent to $\left(\mathscr{N}_{X, B C}^{n-1}\right)^{*} \subset \mathscr{E}_{X, A}^{1}$ since $\mathscr{E}_{X, A}^{1}$ is closed. We adapt the proof of Lemme 1.4 in [La1] to our situation.

Let $\{\theta\} \in H_{A}^{1,1}(X, \mathbb{R})$ be an Aeppli cohomology class such that $(\{\theta\}, \gamma) \geq$ $0, \forall \gamma \in \mathscr{N}_{X, B C}^{n-1}$, and $\theta \in \mathscr{C}_{1,1}^{\infty}(X, \mathbb{R})$ a representative. In particular, $\left(\theta, \omega^{n-1}\right) \geq 0, \forall \omega^{n-1} \in V$, where $V \subset \mathscr{C}_{n-1, n-1}^{\infty}(X, \mathbb{R})$ is the cone of all balanced metrics on $X$. Assume there exists $\omega_{0}^{n-1} \in V$ such that $\left(\theta, \omega_{0}^{n-1}\right)=0$. Let $\beta^{n-1} \in \mathscr{C}_{n-1, n-1}^{\infty}(X, \mathbb{R})$ be a $d$-closed $(n-1, n-1)$ form on $X$. Set $\omega_{t}^{n-1}=(1-t) \omega_{0}^{n-1}+t \beta^{n-1}$ and $f(t)=\left(\theta, \omega_{t}^{n-1}\right)$. Then, there exists $\varepsilon>0$ such that $\omega_{t}^{n-1} \in V$ for $-\varepsilon \leq t \leq \varepsilon$. Therefore $f(-\varepsilon) \geq 0, f(\varepsilon) \geq 0, f(0)=0$, and it follows that $f \equiv 0$, and so $\left(\theta, \beta^{n-1}\right)=0, \forall \beta^{n-1} \in \mathscr{C}_{n-1, n-1}^{\infty}(X, \mathbb{R})$, with $d \beta^{n-1}=0$. The duality between $H_{B C}^{n-1, n-1}(X, \mathbb{R})$ and $H_{A}^{1,1}(X, \mathbb{R})$ implies that $\{\theta\}=0 \in \mathscr{E}_{X, A}^{1}$.

We can suppose now that $\left(\theta, \omega^{n-1}\right)>0, \forall \omega^{n-1} \in V$. Set

$$
\begin{aligned}
& U=\left\{\lambda^{n-1} \in \mathscr{C}_{n-1, n-1}^{\infty}(X, \mathbb{R}) \mid \lambda^{n-1}>0\right\} \\
& E=\left\{\lambda^{n-1} \in \mathscr{C}_{n-1, n-1}^{\infty}(X, \mathbb{R}) \mid d \lambda^{n-1}=0\right\} \\
& F=\left\{\lambda^{n-1} \in E \mid\left(\theta, \lambda^{n-1}\right)=0\right\} .
\end{aligned}
$$

Then $U \cap E=V$ and $V \cap F=\emptyset$, and hence $U \cap F=\emptyset$. By the Hahn-Banach theorem, we can separate $U$ and $F$ by a current $T$ of bidegree $(1,1)$ which is strictly positive on $U$ and vanishes on $F$. Then $T$ is a positive current. Let $\omega^{n-1} \in V$ and define $\lambda=\frac{\left(\theta, \omega^{n-1}\right)}{\left(T, \omega^{n-1}\right)}$. Then $\theta-\lambda T$ is zero on $E$ and from the duality between $H_{A}^{1,1}(X, \mathbb{R})$ and $H_{B C}^{n-1, n-1}(X, \mathbb{R})$ it follows that there exists $S$ a $(1,0)$-current on $X$ such that

$$
\theta-\lambda T=-\bar{\partial} S-\partial \bar{S} .
$$

Hence, the current $\theta+\bar{\partial} S+\partial \bar{S}$ is positive and $\{\theta\} \in \mathscr{E}_{X, A}^{1}$.

Let

$$
\mathscr{K}_{X}=\left\{[\omega] \in H_{B C}^{1,1}(X, \mathbb{R}) \mid \omega \text { is a Kähler metric }\right\}
$$

denote the Kähler cone of $X$. Similarly, we define the balanced cone:

$$
\mathscr{B}_{X}=\left\{[\omega] \in H_{B C}^{n-1, n-1}(X, \mathbb{R}) \mid \omega^{n-1} \text { is a balanced metric }\right\} .
$$

Lemma 2.5. Let $X$ be a compact complex manifold of dimension $n$. 
i) If $X$ is Kähler, then $\mathscr{N}_{X, B C}^{1}=\overline{\mathscr{K}}_{X}$. Moreover, $\mathscr{E}_{X, A}^{n-1}$ is closed and we have, $\mathscr{N}_{X, A}^{n-1} \subset \mathscr{E}_{X, A}^{n-1}$.

ii) If $X$ is balanced, then $\mathscr{N}_{X, B C}^{n-1}=\overline{\mathscr{B}}_{X}$. Moreover, $\mathscr{E}_{X, A}^{1}$ is closed.

Proof. The proof is an adaptation of the arguments in Lemma 2.2.

i) Since $\mathscr{K}_{X} \subset \mathscr{N}_{X, B C}^{1}$ and $\mathscr{N}_{X, B C}^{1}$ is closed, we can see that $\overline{\mathscr{K}}_{X} \subset \mathscr{N}_{X, B C}^{1}$. Conversely, fix $\omega$ a Kähler metric and let $\eta \in \mathscr{N}_{X, B C}^{1}$. Then $\eta+t[\omega] \in \mathscr{K}_{X}$ for any $t>0$ and $\eta=\lim _{t \rightarrow 0} \eta+t[\omega] \in \overline{\mathscr{K}}_{X}$. This proves that $\overline{\mathscr{K}}_{X}=\mathscr{N}_{X, B C}^{1}$.

As in the proof of Lemma 2.2, we show that $\mathscr{E}_{X, A}^{n-1}$ is closed and $\mathscr{N}_{X, A}^{n-1} \subset$ $\mathscr{E}_{X, A}^{n-1}$. Let $\omega$ be a Kähler metric on $X$ and $\eta \in \overline{\mathscr{E}_{X, A}^{n-1}}$. Let $T_{j}$ positive $i \partial \bar{\partial}$-closed currents of bidegree $(n-1, n-1)$ such that $\left\{T_{j}\right\} \rightarrow \eta$ in $H_{A}^{n-1, n-1}(X, \mathbb{R})$. Then the sequence $\left(\int_{X} T_{j} \wedge \omega\right)_{j}$ is bounded, hence we can extract a subsequence $\left(T_{j_{k}}\right)_{k}$ which is weakly convergent to a positive $i \partial \bar{\partial}$-closed current $T$ and $T \in \eta$. Therefore we have $\eta \in \mathscr{E}_{X, A}^{n-1}$. In order to prove the inclusion $\mathscr{N}_{X, A}^{n-1} \subset \mathscr{E}_{X, A}^{n-1}$, let $\eta \in \mathscr{N}_{X, A}^{n-1}$. Then, by definition, $\eta+\varepsilon\left\{\omega^{n-1}\right\} \in \mathscr{E}_{X, A}^{n-1}$, and since $\mathscr{E}_{X, A}^{n-1}$ is closed, it follows that $\eta=\lim _{\varepsilon \rightarrow 0} \eta+\varepsilon\left\{\omega^{n-1}\right\} \in \mathscr{E}_{X, A}^{n-1}$.

ii) We have $\mathscr{B}_{X} \subset \mathscr{N}_{X, B C}^{n-1}$ and, since $\mathscr{N}_{X, B C}^{n-1}$ is closed, it follows that $\overline{\mathscr{B}}_{X} \subset$ $\mathscr{N}_{X, B C}^{n-1}$. Conversely, fix $\omega^{n-1}$ a balanced metric on $X$ and let $\eta \in \mathscr{N}_{X, B C}^{n-1}$. Then $\eta+t\left[\omega^{n-1}\right] \in \mathscr{B}_{X}$ for any $t>0$ and therefore $\eta=\lim _{t \rightarrow 0} \eta+t\left[\omega^{n-1}\right] \in$ $\overline{\mathscr{B}}_{X}$.

We show next that $\mathscr{E}_{X, A}^{1}$ is closed. Let $\omega^{n-1}$ be a fixed balanced metric on $X$ and consider a sequence $\left(S_{j}\right)_{j}$ of positive $i \partial \bar{\partial}$-closed currents of bidegree $(1,1)$ converging to $\eta \in H_{A}^{1,1}(X, \mathbb{R})$. Then the sequence $\left(\int_{X} S_{j} \wedge \omega^{n-1}\right)_{j}$ is bounded and so there exists a subsequence $\left(S_{j_{k}}\right)_{k}$ converging weakly to a positive $i \partial \bar{\partial}$-closed current $S$ of bidegree $(1,1)$. That means $\{S\}=\eta \in$ $\mathscr{E}_{X, A}^{1}$, and so the cone $\mathscr{E}_{X, A}^{1}$ is closed.

Remark 2.6. If $X$ is a Kähler manifold, there exists a natural map $\varpi: \mathscr{K} \rightarrow \mathscr{B}$ given by $\varpi([\omega])=\left[\omega^{n-1}\right]$. Fu and Xiao $[\mathrm{FX}]$ showed that the map $p$ is injective [FX, Proposition 1.1]. Moreover, $p$ is not always surjective. More precisely, they provided examples of manifolds [FX, pages 11 and 12] where $\mathscr{B}_{X} \backslash \varpi\left(\mathscr{K}_{X}\right) \neq \emptyset$.

We have natural morphisms

$$
j_{1}: H_{B C}^{1,1}(X, \mathbb{R}) \rightarrow H_{A}^{1,1}(X, \mathbb{R})
$$

and

$$
j_{n-1}: H_{B C}^{n-1, n-1}(X, \mathbb{R}) \rightarrow H_{A}^{n-1, n-1}(X, \mathbb{R})
$$

which are isomorphisms if $X$ is Kähler, due to the $\partial \bar{\partial}$-lemma.

Proposition 2.7. Let $X$ be a compact Kähler manifold of dimension $n$. Then

$$
j_{n-1}\left(\mathscr{E}_{X, B C}^{n-1}\right)=\mathscr{E}_{X, A}^{n-1}
$$

and

$$
j_{1}\left(\mathscr{N}_{X, B C}^{1}\right)=\mathscr{N}_{X, A}^{1}
$$


Proof. The second statement follows from the first one by duality. From Theorem 2.4 , we have that $\left(\mathscr{N}_{X, B C}^{1}\right)^{*}=\mathscr{E}_{X, A}^{n-1}$ since $\mathscr{E}_{X, A}^{n-1}$ is closed. Corollary 0.3 in [DP] implies that the currents of the form $j_{n-1}\left(\left[\int_{Y} \omega^{p-1} \wedge \bullet\right]\right)$, where $Y$ is a $p$-dimensional analytic subset of $X$ and $\omega$ is a Kähler metric on $X$, generate the cone $\mathscr{E}_{X, A}^{n-1}$. Since the currents $\left(\int_{Y} \omega^{p-1} \wedge \bullet\right)$ are $d$-closed and positive, we see that $j_{n-1}\left(\mathscr{E}_{X, B C}^{n-1}\right)=$ $\mathscr{E}_{X, A}^{n-1}$.

Remark 2.8. Given a compact complex Kähler manifold of dimension $n$, Conjecture 2.3 in $[\mathrm{BDPP}]$ implies that $j_{n-1}\left(\mathscr{N}_{X, B C}^{n-1}\right)=\mathscr{N}_{X, A}^{n-1}$, i.e., that the dual of the pseudoeffective cone $\mathscr{E}_{X, B C}^{1}$ is the closure of the cone of classes of balanced metrics.

2.1. Néron-Severi groups. For a compact complex manifold $X$ of dimension $n$ we have natural maps

$$
\begin{gathered}
\alpha_{p}: H_{B C}^{p, p}(X, \mathbb{R}) \rightarrow H_{d R}^{2 p}(X, \mathbb{R}), \\
\beta_{p}: H_{d R}^{2 p}(X, \mathbb{R}) \rightarrow H_{A}^{p, p}(X, \mathbb{R}), \\
\gamma_{p}: H^{2 p}(X, \mathbb{Z}) \rightarrow H_{d R}^{2 p}(X, \mathbb{R}) .
\end{gathered}
$$

Define the Néron-Severi groups

$$
H_{B C, N S}^{p, p}(X, \mathbb{R})=\alpha_{p}^{-1}\left(\gamma_{p}\left(H^{2 p}(X, \mathbb{Z})\right)\right) \otimes_{\mathbb{Z}} \mathbb{R} \subset H_{B C}^{p, p}(X, \mathbb{R})
$$

and

$$
H_{A, N S}^{p, p}(X, \mathbb{R})=\beta_{p}\left(\gamma_{p}\left(H^{2 p}(X, \mathbb{Z})\right)\right) \otimes_{\mathbb{Z}} \mathbb{R} \subset H_{A}^{p, p}(X, \mathbb{R}) .
$$

If $X$ is projective, then the canonical morphisms

$$
H_{B C, N S}^{1,1}(X, \mathbb{R}) \rightarrow H_{A, N S}^{1,1}(X, \mathbb{R})
$$

and

$$
H_{B C, N S}^{n-1, n-1}(X, \mathbb{R}) \rightarrow H_{A, N S}^{n-1, n-1}(X, \mathbb{R}) .
$$

are isomorphisms, and the standard notation for these groups are $N^{1}$ or $N S_{X}^{1}$, and $N_{1}$, respectively. The group $N S_{X}^{1}$ is generated by classes of divisors on $X$, and by the Hard Lefschetz Theorem, it follows that $N_{1}$ is generated by classes of curves on $X$.

Let the subscript $N S$ denote the intersection of a cone (nef or pseudoeffective) with the Néron-Severi groups.

Proposition 2.9. If $X$ is compact Kähler of dimension n, then the pairing

$$
H_{B C, N S}^{p, p}(X, \mathbb{R}) \times H_{A, N S}^{n-p, n-p}(X, \mathbb{R}) \rightarrow \mathbb{R},([\alpha],\{\beta\}) \rightarrow \int_{X} \alpha \wedge \beta
$$

is nondegenerate and all the equalities of Theorem 2.4 hold at the Néron-Severi level. Moreover,

and

$$
j_{n-1}\left(\mathscr{E}_{B C, N S}^{n-1}\right)=\mathscr{E}_{A, N S}^{n-1}
$$

If $X$ is projective, then

$$
j_{1}\left(\mathscr{N}_{B C, N S}^{1}\right)=\mathscr{N}_{A, N S}^{1} .
$$

$$
j_{n-1}\left(\mathscr{N}_{B C, N S}^{n-1}\right)=\mathscr{N}_{A, N S}^{n-1}
$$

and

$$
j_{1}\left(\mathscr{E}_{B C, N S}^{1}\right)=\mathscr{E}_{A, N S}^{1}
$$


Proof. The only non-trivial statement is (2.8), as (2.7) follows by duality.

Let $\{T\} \in \mathscr{E}_{A, N S}^{1}$ where $T$ is a positive, $\partial \bar{\partial}$-closed current, and let $j_{1}([S])=\{T\}$, $[S] \in H_{B C, N S}^{1,1}(X, \mathbb{R})$. We want to show that $[S] \in \mathscr{E}_{B C, N S}^{1}$. For the proof, we follow $[\mathrm{To}]$.

From [BDPP, Theorem 2.2] we see that it is enough to check that

$$
\left([S],\left\{p_{*}\left(A_{1} \cap \ldots \cap A_{n-1}\right)\right\}\right) \geq 0,
$$

where $p: Y \rightarrow X$ is a proper modification of $X$ and $A_{1}, \ldots, A_{n-1}$ are very ample line bundles on $Y$. However, from Theorem 3 in [AB2], there exists $T^{\prime}$, a positive pluriharmonic current on $Y$ which is the total transform of $T$, and we have

$$
\begin{aligned}
\left([S],\left\{p_{*}\left(A_{1} \cap \ldots \cap A_{n-1}\right)\right\}\right) & =\left(\{T\},\left[p_{*}\left(A_{1} \cap \ldots \cap A_{n-1}\right]\right)\right. \\
& =\left(T^{\prime}, A_{1} \cap \ldots \cap A_{n-1}\right) \\
& \geq 0 .
\end{aligned}
$$

Remark 2.10. Boucksom, Demailly, Păun and Peternell define [BDPP, Definition 1.1] the pseudoeffective cone $\mathscr{E}_{N S}$ as $\mathscr{E}_{X, d R}^{1} \cap N S_{\mathbb{R}}(X)$, where

$$
N S_{\mathbb{R}}(X)=\left(H_{\mathbb{R}}^{1,1}(X) \cap H^{2}(X, \mathbb{Z}) / \text { torsion }\right) \otimes_{\mathbb{Z}} \mathbb{R} .
$$

Formula (2.8) above implies in particular that, at the Néron-Severi level, the pseudoeffective cones $\mathscr{E}_{B C, N S}, \mathscr{E}_{A, N S}$ and $\mathscr{E}_{N S}$ coincide via the canonical isomorphisms between the cohomology groups $H_{B C, N S}^{1,1}(X, \mathbb{R}), H_{A, N S}^{1,1}(X, \mathbb{R})$, and $N S_{\mathbb{R}}(X)$.

\section{UNIRULED MANIFOLDS AND BALANCED METRICS}

3.1. Bimeromorphism invariance. We prove here that the existence of a balanced metric of positive total scalar Chern curvature is an invariant property under bimeromorphisms.

Proof of Theorem A. By [AKMW], we can assume that $p: Y \rightarrow X$ is a blowup with smooth center $C$ and let $E$ be the exceptional divisor of $p$. Then $K_{Y}=$ $p^{*} K_{X}+a E$, where $a=\operatorname{codim}_{X} C-1>0$.

Suppose first that $X$ admits a balanced metric $\omega_{X}^{n-1}$ which is negative on the canonical line bundle of $X$. Let $i: E \rightarrow Y$ denote the inclusion. Since

$$
\int_{Y} c_{1}(E) \wedge p^{*} \omega_{X}^{n-1}=\int_{E} i^{*} p^{*} \omega_{X}^{n-1}=\int_{C} \omega_{X}^{n-1}=0
$$

we find that

$$
\int_{Y} c_{1}\left(K_{Y}\right) \wedge p^{*} \omega_{X}^{n-1}=\int_{Y} c_{1}\left(p^{*} K_{X}\right) \wedge p^{*} \omega_{X}^{n-1}=\int_{X} c_{1}\left(K_{X}\right) \wedge \omega_{X}^{n-1}<0 .
$$

It is known that $Y$ is also balanced [AB3], and if $\omega_{Y}^{n-1}$ is a balanced metric on $Y$, then $p^{*} \omega_{X}^{n-1}+\varepsilon \omega_{Y}^{n-1}$ is a balanced metric and

$$
\int_{Y} c_{1}\left(K_{Y}\right) \wedge\left(p^{*} \omega_{X}^{n-1}+\varepsilon \omega_{Y}^{n-1}\right)<0
$$

for a small $\varepsilon>0$. 
Conversely, suppose that $Y$ supports a balanced metric $\omega_{Y}^{n-1}$ such that

$$
\int_{Y} c_{1}\left(K_{Y}\right) \wedge \omega_{Y}^{n-1}<0
$$

and suppose that

$$
\int_{X} c_{1}\left(K_{X}\right) \wedge \omega^{n-1} \geq 0
$$

for any balanced metric $\omega^{n-1}$ on $X$. Then

$$
\int_{X} c_{1}\left(K_{X}\right) \wedge \eta \geq 0
$$

for any class $[\eta] \in \mathscr{N}_{B C, X}^{n-1}$. Therefore, by Theorem $\left.2.4 \mathrm{iv}\right),\left\{c_{1}\left(K_{X}\right)\right\} \in \mathscr{E}_{X, A}^{1}$, i.e., there exists $T$ a positive $\partial \bar{\partial}$-closed $(1,1)$-current in the Aeppli cohomology class $\left\{c_{1}\left(K_{X}\right)\right\}$. From [AB2], it follows that there exists a positive $\partial \bar{\partial}$-closed current on $Y$ denoted by $T^{\prime}$, which is the total transform of $T$. This means that

$$
T^{\prime} \in\left\{c_{1}\left(p^{*} K_{X}\right)\right\}=p^{*}\left\{c_{1}\left(K_{X}\right)\right\}
$$

In particular, $\left\{c_{1}\left(p^{*} K_{X}\right)\right\} \in \mathscr{E}_{Y, A}^{1}$ and therefore

$$
\left\{c_{1}\left(K_{Y}\right)\right\}=\left\{c_{1}\left(p^{*} K_{X}\right)\right\}+a\{[E]\} \in \mathscr{E}_{Y, A}^{1}
$$

which contradicts (3.1).

3.2. Metrics on Mori fiber spaces. We start by recalling background definitions from the minimal model program.

Definition 3.1. A compact complex variety $Y$ is called $\mathbb{Q}$-factorial if every Weil divisor of $Y$ is $\mathbb{Q}$-Cartier.

Let $Y$ be normal variety such that $m K_{Y}$ is Cartier for some $m>0$, and let $f: Z \rightarrow Y$ be a resolution of singularities. Up to numerical equivalence, we can write

$$
K_{Z} \equiv{ }_{\mathbb{Q}} f^{*}\left(K_{Y}\right)+\sum_{i} a_{i} E_{i}
$$

where the $E_{i}$ 's are the $f$-exceptional divisors, and $a_{i} \in \mathbb{Q}$.

Definition 3.2. We say that $Y$ has log-terminal singularities if $a_{i}>-1$, for all $i$.

It is well-known that this definition is independent of the choice of the resolution $[\mathrm{KM}]$.

Definition 3.3. A normal compact complex variety $Y$ with only $\mathbb{Q}$-factorial logterminal singularities equipped with a map $\phi: Y \rightarrow B$ is called a Mori fiber space if the following conditions are satisfied:

i) The map $\phi$ is a morphism with connected fibers onto a normal variety $B$ with $\operatorname{dim} B<\operatorname{dim} Y$.

ii) All the curves $C$ in the fibers of $\phi$ are numerically proportional and $K_{Y} \cdot C<$ 0 . 
3.2.1. The projective case. We give here a first proof of Theorem B based on the the minimal model program. A second proof, circumventing the minimal model program follows.

Proposition 3.1. Let $\phi: Y \rightarrow B$ be a Mori fiber space, with $Y$ and $B$ projective. Then, there exists an ample line bundle $H$ on $Y$ such that

$$
K_{Y} \cdot H_{Y}^{n-1}<0 .
$$

Proof. If $\operatorname{dim} B=0$, by Kleiman's Ampleness Criterion $-K_{Y}$ is ample, and so $K_{Y} \cdot H^{n-1}<0$ for all ample line bundles on $Y$.

Assume now that $\operatorname{dim} B=b>0$ and fix an ample line bundle $L$ on $B$, and $H_{0}$ an ample line bundle on $Y$. Let

$$
H_{m}=m \phi^{*} L+H_{0} .
$$

Then $H_{m}$ is an ample line bundle on $Y$ for all $m>0$, and

$$
\begin{aligned}
K_{Y} \cdot H_{m}^{n-1} & =K_{Y} \cdot\left(m \phi^{*} L+H_{0}\right)^{n-1} \\
& =c(n, b) m^{b} K_{Y} \cdot\left(\phi^{*} L\right)^{b} \cdot H_{0}^{n-1-b}+O\left(m^{b-1}\right) \\
& =c(n, b) m^{b}\left(L^{b}\right)\left(K_{F} \cdot H_{0}^{n-1-b}\right)+O\left(m^{b-1}\right),
\end{aligned}
$$

where $c(n, b)$ is a positive integer depending only on $n$ and $b$, and $F$ denotes the fiber of $\phi$. By the relative version of Kleiman's Ampleness Criterion [KM, Theorem 1.44] we see that $-K_{F}$ is ample, and so $K_{Y} \cdot H_{m}^{n-1}<0$ for $m \gg 0$. Take now $H_{Y}=H_{m}$ for some fixed $m \gg 0$.

The first proof of Theorem B. Let $X$ be a smooth, Moishezon, uniruled manifold of dimension $n>0$. Then there exists a smooth projective manifold $Y$ of dimension $n$ bimeromorphic to $X$. Since uniruledness is preserved under bimeromorphic transformations, $Y$ is uniruled. According to [Ko, Theorem IV.1.9], there exists a non-constant holomorphic map $u: \mathbb{P}_{1} \rightarrow Y$, such that $u^{*} T_{Y}$ is globally generated. Since we have an injection from $\mathscr{O}_{\mathbb{P}^{1}}(2)=T_{\mathbb{P}^{1}}$ to $u^{*} T_{Y}$, it follows that $\operatorname{deg} u^{*} T_{Y} \geq 2$, and so $K_{Y} \cdot u\left(\mathbb{P}_{1}\right)<0$. But the curve $u\left(\mathbb{P}_{1}\right)$ moves in a family covering $X$, and so by [BDPP, Theorem 0.2], the canonical bundle $K_{Y}$ is not pseudoeffective. This implies, according to [BCHM, Corollary 1.3.3], that $Y$ is birational to a Mori fiber space $\phi: Z \rightarrow B$ with $Z$ and $B$ projective. In general, $Z$ is not smooth, and let $f: \hat{Z} \rightarrow Z$ be a desingularization. Then, there exists an ample line bundle $H_{\hat{Z}}$ on $\hat{Z}$ such that

$$
K_{\hat{Z}} \cdot H_{\hat{Z}}^{n-1}<0
$$

Indeed, from Proposition 3.1, we know that there exists an ample line bundle $H_{Z}$ on $Z$ such that $K_{Z} \cdot H_{Z}^{n-1}<0$. Fix $H_{0}$ be an ample line bundle on $\hat{Z}$. For every $m>0$, let

$$
H_{m}=m f^{*} H_{Z}+H_{0}
$$

Then $H_{m}$ is an ample line bundle on $\hat{Z}$, and

$$
\begin{aligned}
K_{\hat{Z}} \cdot H_{m}^{n-1} & =\left(f^{*} K_{Z}+\sum_{i} a_{i} E_{i}\right) \cdot\left(m f^{*} H_{Z}+H_{0}\right)^{n-1} \\
& =m^{n-1} K_{Z} \cdot H_{Z}^{n-1}+O\left(m^{n-2}\right)<0,
\end{aligned}
$$

for $m$ sufficiently large. Take now $H_{\hat{Z}}=H_{m}$ for fixed $m \gg 0$. Since $H_{\hat{Z}}$ is ample, the first Chern class of $k H_{m}$ is represented by the Kähler form of a Hodge metric 
$\omega_{\hat{Z}}$ for sufficiently large $k$. In particular, we found on $\hat{Z}$ a Kähler metric $\omega$ such that

$$
\int_{\hat{Z}} c_{1}\left(K_{\hat{Z}}\right) \wedge \omega_{\hat{Z}}^{n-1}<0 .
$$

Since $\hat{Z}$ and $X$ are smooth and bimeromorphic manifolds, we can apply now Theorem A to conclude that $X$ admits a balanced metric $\omega^{n-1}$ such that

$$
\int_{X} c_{1}\left(K_{X}\right) \wedge \omega^{n-1}<0
$$

We give next a short second proof of Theorem B using the results presented in Section 2. In fact, for projective manifolds we can prove a slightly more precise result:

Proposition 3.2. Let $X$ be a uniruled projective manifold of dimension $n$. Then there exists $\omega^{n-1}$ a balanced metric, $\left[\omega^{n-1}\right] \in H_{B C, N S}^{n-1, n-1}(X, \mathbb{R})$ such that

$$
\int_{X} c_{1}\left(K_{X}\right) \wedge \omega^{n-1}<0
$$

Proof. Since $X$ is uniruled, arguing as in the first proof of Theorem B, we see that the canonical bundle $K_{X}$ is not pseudoeffective. Hence, as in Remark 2.10, $c_{1}\left(K_{X}\right) \notin \mathscr{E}_{B C, N S}^{1}$. As a consequence, from Proposition 2.9 we see $c_{1}\left(K_{X}\right) \notin \mathscr{E}_{A, N S}^{1}$. Theorem 2.4 now implies the existence of a balanced metric $\omega^{n-1}$ with integral class whose pairing with $c_{1}\left(K_{X}\right)$ is negative.

The proof of Theorem B now follows from Proposition 3.2 and Theorem A.

3.2.2. The Kähler case. The first proof of Therem B can be adapted in Kähler setting. ${ }^{3}$

Proposition 3.3. Let $\phi: Z \rightarrow S$ be a Mori fiber space where $Z$ and $S$ are Kähler spaces. Then there exists a Kähler form $\eta$ on $Z$ such that

$$
K_{Z} \cdot\left[\eta^{n-1}\right]<0 \text {. }
$$

Proof. Fix $\omega_{S}$ and $\omega_{Z}$ Kähler forms on $S$ and $Z$, respectively and consider the family of Kähler forms

$$
\eta_{t}=t \phi^{*} \omega_{S}+\omega_{Z}, t>0 .
$$

As in the proof of Proposition 3.1 we see that $K_{Z} \cdot\left[\eta_{t}^{n-1}\right]<0$ for $t \gg 0$. We omit the details.

Proof of Theorem $C$. Let $X$ be a smooth, uniruled, 3-dimensional manifold of class $\mathscr{C}$. That means there exists a uniruled, 3 -dimensional, Kähler manifold $Y$ bimeromorphic to $X$.

According to Höring and Peternell [HP2, Theorem 1.1], $Y$ is bimeromorphic to a Kähler Mori fiber space $Z$ as in Proposition 3.3. In general, $Z$ is not smooth. Let

\footnotetext{
${ }^{3}$ Here we have to work with singular Kähler spaces. For the basic notions in the theory of a Kähler space we refer the interested reader to the sections 2 and 3 in [HP2].
} 
$f: \hat{Z} \rightarrow Z$ be a desingularization. By [Va, 1.3.1], $\hat{Z}$ is a smooth Kähler manifold. As in the first proof of Theorem B, we can find a Kähler metric $\omega$ on $\hat{Z}$ such that

$$
\int_{Y} c_{1}\left(K_{\hat{Z}}\right) \wedge \omega^{2}<0
$$

Applying now Theorem A, we can conclude that $X$ admits a balanced metric with the property claimed in Theorem C.

3.3. Characterization of uniruledness. In this very short section, we complete the characterization of uniruledness, by proving a converse to Theorems B and C.

Proof of Theorem D. The implication ii) $\Longrightarrow$ iii) is the content of Theorems B and $\mathrm{C}$, while $i i i) \Longrightarrow i v$ ) is trivial. Morever, from the positivity criterion of Lamari [La1, Théorème $1.2(1)$ ] (see also Theorem 2.4, part i)) we can see that $i v) \Longrightarrow i$ ).

Finally, it remains to show that $i) \Longrightarrow i i$ ). Since neither uniruledness nor the pseudoeffectivity of the canonical divisor is affected by bimeromorphic transformations, we may assume that either $X$ is projective, or $X$ is a non-projective Kähler threefold. In the first case, the remarkable Corollary 0.3 in [BDPP] shows that $X$ is uniruled, while in the second case we reach the same conclusion using the equally remarkable Corollary 1.2 in $[\mathrm{Br}]$.

\section{BALANCED METRICS ON TWISTOR SPACES}

A large class of examples of uniruled complex manifolds is provided by the manifolds bimeromorphic to the twistor spaces of closed anti-self dual four-manifolds. These are compact complex manifolds of dimension three [AHS], equipped with a one-parameter family of balanced metrics $[\mathrm{Mi}, \mathrm{Mu}]$. In this section, we show that among these metrics there exists a balanced metric of positive total Chern scalar curvature.

We start by recalling the construction of the twistors spaces.

Let $(M, g)$ be an oriented Riemannian 4-manifold. Under the action of the Hodge $\star$-operator

$$
\star: \Lambda^{2} M \rightarrow \Lambda^{2} M,
$$

one has a decomposition $\Lambda^{2} M=\Lambda_{+} \oplus \Lambda_{-}$into self-dual and anti-self-dual forms, corresponding to the $( \pm 1)$ - eigenvalues of $\star$.

Let $\mathscr{R}: \Lambda^{2} \rightarrow \Lambda^{2}$ be the Riemannian curvature operator. Under the action of $S O(4)$, the Riemannian curvature operator decomposes as

$$
\mathscr{R}=\frac{s}{6} I d+W^{-}+W^{+}+\stackrel{\circ}{r}
$$

where $s$ denotes the scalar curvature, $W^{ \pm}$are the self-dual and anti-self-dual components of the Weyl curvature operator, and $\stackrel{\circ}{r}$ is the trace-free Ricci curvature operator. The oriented Riemannian 4 -manifold $(M, g)$ is said to be anti-self-dual (ASD) if $W^{+}=0$. This definition is conformally invariant, i.e. if $g$ is ASD, so is $a g$ for any smooth positive function $a$.

The twistor space of a conformal Riemannian manifold $(M,[g])$ is the total space of the sphere bundle of the rank three real vector bundle of self-dual 2 -forms $\mathscr{Z}:=S\left(\Lambda_{+}\right)$. Let $\varpi: \mathscr{Z} \rightarrow M$ be the projection onto $M$. For every $x \in M$, the fiber $\varpi^{-1}(x)$ corresponds to the set of $g$-orthogonal complex structures compatible 
with the given orientation. More precisely, any such $j$ defines the unit length selfdual form

$$
\omega(v, w)=\frac{1}{\sqrt{2}} g(v, j w) .
$$

The real six-dimensional manifold $\mathscr{Z}$ comes equipped with an almost complex structure, that is an endomorphism $\mathscr{J}: T \mathscr{Z} \rightarrow T \mathscr{Z}$ satisfying $\mathscr{J}^{2}=-1$. The Levi-Civita connection $\nabla$ of $M$ gives rise to a splitting $T \mathscr{Z}=\mathscr{H} \oplus \mathscr{V}$ of the tangent bundle of $\mathscr{Z}$ into horizontal and vertical components. At a point $(\sigma, x) \in \mathscr{Z}$, the vertical distribution $\mathscr{V}$ consists of the vectors tangent to the fiber of $\varpi$, which is an oriented metric 2 -sphere, and hence equipped with a compatible complex structure $I$. On the other hand, the almost complex structure $j$ associated to $\sigma$ discussed above naturally lifts to the horizontal distribution $\mathscr{H}$. Then, $\mathscr{J}$ is defined as $\mathscr{J}=(j, I)$. A remarkable result of Atiyah, Hitchin and Singer [AHS] asserts that $\mathscr{J}$ is integrable if and only if the metric $g$ is ASD. In such a case, the fibers $\varpi^{-1}(x), x \in M$ are smooth rational curves, and so $\mathscr{Z}$ is uniruled.

We assume from now on that $\mathscr{Z}$ is the twistor space associated to a closed, oriented 4-manifold $M$ equipped with an ASD conformal class $[g]$. We fix $g \in[g]$.

Let $h_{t}$ be the family of Riemannian metrics on $\mathscr{Z}$ defined by

$$
h_{t}=\varpi^{*} g+t g^{\text {vert }},
$$

where $t>0, g$ is the metric of $M$ and $g^{\text {vert }}$ is the restriction of the metric induced on $\Lambda_{+}$to the vertical distribution $\mathscr{V}$. Then $\varpi:\left(\mathscr{Z}, h_{t}\right) \rightarrow(M, g)$ is a Riemannian submersion with totally geodesic fibers. Moreover, the metrics $h_{t}$ are compatible with $\mathscr{J}$. Michelsohn states [Mi, Section 6] the existence of balanced metrics on $\mathscr{Z}$. A proof that the metrics $h_{t}$ are in fact balanced follows from Corollary 3.5 and Lemma 4.1 in $[\mathrm{Mu}]$.

The Riemannian scalar curvature of the metrics $h_{t}$ is computed by Davidov and Muškarov [DM]. More precisely, in [DM, Corollary 4.2] it is proved that for every $(\sigma, x) \in \mathscr{Z}$,

$$
s_{\mathscr{Z}}(\sigma, x)=s_{M}(x)+\frac{t}{4}\left(\|\mathscr{R}(\sigma)\|^{2}-\left\|\mathscr{R}_{-}\right\|_{x}^{2}\right)+\frac{2}{t},
$$

where $s_{\mathscr{Z}}$ and $s_{M}$ denote the scalar curvatures of $\mathscr{Z}$ and $M$, respectively, and $\mathscr{R}_{-}=\frac{s}{12} I d+W^{-}+\stackrel{\circ}{r}$ is the restriction of $\mathscr{R}$ to $\Lambda_{-}$. In particular, for $0<t \ll 1$, we see that the metric $h_{t}$ satisfies $s_{\mathscr{Z}}>0$.

Proof of Theorem E. Let $X$ be a complex manifold bimeromorphic to a twistor space $\mathscr{Z}$.

Let $\omega_{t}$ be the Kähler 2 -form of the balanced metric $h_{t}$ on $\mathscr{Z}$ defined by (4.1). By Corollary 1.2, we have

$$
\int_{\mathscr{Z}} c_{1}(\mathscr{Z}) \wedge \omega_{t}^{2} \geq \frac{1}{12 \pi} \int_{\mathscr{Z}} s_{\mathscr{Z}} \omega_{t}^{3}>0,
$$

for $0<t \ll 1$. The conclusion of Theorem $\mathrm{E}$ now follows from Theorem A.

Remark 4.1. Twistor spaces of class $\mathscr{C}$ are rather scarce. Campana [Ca2], and LeBrun and Poon [LP], independently proved that if the twistor space $\mathscr{Z}$ of an ASD four-manifold $M$ is of Fujiki class $\mathscr{C}$, then $\mathscr{Z}$ is Moishezon and $M$ is homeomorphic to either $S^{4}$ or the connected sum of $n \geq 1$ copies of $\overline{\mathbb{C P}^{2}}$, the complex projective plane endowed with the opposite orientation. However, a result of Taubes [Ta] asserts that every Riemannian manifold $M$ can be equipped with an ASD metric 
after taking the connected sum with sufficiently many copies of $\overline{\mathbb{C P}^{2}}$, hence the twistor spaces provide a large family of balanced manifolds which are not of class $\mathscr{C}$.

Acknowledgements. The first author was supported by the CNCS grant PNII-ID-PCE-2011-3-0269 during the preparation of this work. The second author acknowledges the support of the Simons Foundation's "Collaboration Grant for Mathematicians", while the third author was supported by the NSF grant DMS1309029. The second and third author would like to thank IHÉS for hospitality, while this project was finalized. The authors are grateful to the anonymous referees for many useful comments which helped us improve this article.

\section{REFERENCES}

[AKMW] D. Abramovich, K. Karu, K. Matsuki, J. Włodarczyk, Torification and factorization of birational maps. J. Amer. Math. Soc. 15 (2002), no. 3, 531-572.

[AB1] L. Alessandrini, G. Bassanelli, Metric properties of manifolds bimeromorphic to compact Kähler spaces. J. Diff. Geom. 37 (1993) 95-121.

[AB2] L. Alessandrini, G. Bassanelli, Modifications of compact balanced manifolds. C.R. Acad. Sci. Paris Math. 320 (1995), 1517-1522.

[AB3] L. Alessandrini, G. Bassanelli, The class of compact balanced manifolds is invariant under modifications. Complex analysis and geometry (Trento, 1993), 1-17, Lecture Notes in Pure and Appl. Math., 173, Dekker, New York, 1996.

[AHS] M.F. Atiyah, N.J. Hitchin, I.M. Singer, Self-duality in four-dimensional Riemannian geometry. Proc. Roy. Soc. London Ser. A 362 (1978), no. 1711, 425-461.

[BCHM] C. Birkar, P. Cascini, C. D. Hacon, J. McKernan, Existence of minimal models for varieties of log general type. J. Amer. Math. Soc. 23 (2010), no. 2, 405-468.

[BDPP] S. Boucksom, J.-P. Demailly, M. Păun, T. Peternell, The pseudoeffective cone of a compact Kähler manifold and varieties of negative Kodaira dimension. J. Algebraic Geom. 22 (2013), 201-248.

[Br] M. Brunella, A positivity property for foliations on compact Kähler manifolds. Intern. J. Math. 17 (2006), 35-43.

[Ca2] F. Campana, The class $\mathscr{C}$ is not stable by small deformations. Math. Ann. 290 (1991), no. $1,19-30$.

[DM] J. Davidov, O. Muškarov, On the Riemannian curvature of a twistor space. Acta Math. Hungar. 58 (1991), no. 3-4, 319-332.

[DP] J.-P. Demailly, M. Păun, Numerical characterization of the Kähler cone of a compact Kähler manifold. Ann. of Math. (2) 159 (2004), no. 3, 1247-1274.

[De] J.-P. Demailly, Regularization of closed positive currents and intersection theory. J. Algebraic Geom. 1 (1992), no. 3, 361-409.

[DPS] J.-P. Demailly, T. Peternell, M. Schneider, Holomorphic line bundles with partially vanishing cohomology. Proceedings of the Hirzebruch 65 Conference on Algebraic Geometry (Ramat Gan, 1993), 165-198, Israel Math. Conf. Proc., 9, Bar-Ilan Univ., Ramat Gan, 1996.

[Fr] R. Friedman, Simultaneous resolution of threefold double points. Math. Ann., 274 (4) (1986), 671-689.

[FLY] J. Fu, J. Li, S.-T. Yau, Balanced metrics on non-Kähler Calabi-Yau threefolds. J. Diff. Geom. 90 (2012), 81-130.

[FX] J. Fu, J. Xiao, Relations between the Kähler cone and the balanced cone of a Kähler manifold. arXiv:1203.2978v1 [math.DG]

[Ga1] P. Gauduchon, Le théorème de l'excentricité nulle. C.R Acad. Sci. Paris Sér. A-B 2285 (1977), no.5, A387-A390.

[Ga2] P. Gauduchon, La 1-forme de torsion d'une variété hermitienne compacte. Math. Ann. 267 (1984), no. 4, 495-518.

[HW] G. Heier, B. Wong, Scalar curvature and uniruledness on projective manifolds. Comm. Anal. Geom. 20 (2012), no. 4, 751-764. 
[Hi] N. J. Hitchin, Kählerian twistor spaces. Proc. London Math. Soc. (3) 43 (1981), 133150.

[HP1] A. Höring, T. Peternell Minimal models for Kähler thereefolds. arXiv:1304.4013v1 [math.AG]

[HP2] A. Höring, T. Peternell, Mori fibre spaces for Kähler thereefolds. arXiv:1310.5837v1 [math.AG]

[KS] K. Kodaira, D. C. Spencer, On deformations of complex analytic structures, III. Stability theorems for complex structures, Ann. of Math. (2), 71, no. 1 (1960), 43-76.

[Ko] J. Kollár, Rational curves on algebraic varieties. Ergebnisse der Mathematik und ihrer Grenzgebiete. 3. Folge. A Series of Modern Surveys in Mathematics [Results in Mathematics and Related Areas. 3rd Series. A Series of Modern Surveys in Mathematics], 32. Springer-Verlag, Berlin, 1996.

[KM] J. Kollár, S. Mori, Birational geometry of algebraic varieties. Cambridge Tracts in Mathematics, Vol. 134, Cambridge University Press, Cambridge, 1998.

[La1] A. Lamari, Le cône kählérien d'une surface, J. Math. Pures Appl., 78 (1999), 249-263.

[La2] A. Lamari, Courants kählériens et surfaces compactes. Ann. Inst. Fourier, 49 no. 1 (1999), 263-285.

[LP] C. LeBrun, Y.S Poon, Twistors, Khler manifolds, and bimeromorphic geometry. II. J. Amer. Math. Soc. 5 (1992), no. 2, 317-325.

[Le] P. Lelong, Intégration sur un ensemble analytique complexe. Bull. Soc. Math. France 85 (1957), 239-262.

[LY] K. Liu, X. Yang, Ricci Curvatures on Hermitian manifolds. arXiv:1404.2481v2 [math.DG].

[LT] P. Lu, G. Tian, The complex structures on connected sums of $S^{3} \times S^{3}$. Manifolds and geometry (Pisa, 1993), 284-293, Sympos. Math., XXXVI, Cambridge Univ. Press, Cambridge, 1996.

[Mi] M. L. Michelsohn, On the existence of special metrics in complex geometry. Acta Math., 149, Number 1 (1982), 261-295.

[Mu] O. Muşkarov, Almost Hermitian structures on twistor spaces and their types. Atti del Seminario Matematico e Fisico dell'Universitá di Modena 37, no. 2 (1989). 285-297.

[Ră] R. Răsdeaconu, On rationally connected threefolds. http://www.math.vanderbilt.edu/ $\sim$ rasdear/weakratcon.pdf.

[Sc] M. Schweitzer, Autour de la cohomologie de Bott-Chern. arXiv:0709.3528 [math.AG].

[Ta] C.H. Taubes, The existence of anti-self-dual conformal structures. J. Differential Geom. 36 (1992), no. 1, 163-253.

[To] M. Toma, A note on the cone of mobile curves. C. R. Math. Acad. Sci. Paris 348 (2010), 71-73.

[Va] J. Varouchas, Kähler spaces and proper open morphisms. Math. Ann. 283 (1989), no. $1,13-52$.

[Ya] S. T. Yau, On the curvature of compact Hermitian manifolds. Invent. Math. 25 (1974), pp. 213-239.

Institute of Mathematics of the Romanian Academy, P.O. Box 1-764, Bucharest 014700, ROMANIA

E-mail address: Ionut.Chiose@imar.ro

Department of Mathematics, 1326 Stevenson Center, Vanderbilt University, Nashville, TN, 37240, USA

E-mail address: rares.rasdeaconu@vanderbilt.edu

Department of Mathematics, 1326 Stevenson Center, Vanderbilt University, Nashville, TN, 37240, USA

E-mail address: ioana.suvaina@vanderbilt.edu 